Research Article

\title{
Some results on an encryption method using subset-sums of pseudo-recursive sequences
}

\author{
Bence Bakos*, Máté Pálfy \\ Institute of Mathematics, Eötvös Loránd University, H-1117 Pázmány st. 1/c, Budapest, Hungary
}

(Received: 27 February 2021. Received in revised form: 24 March 2021. Accepted: 25 March 2021. Published online: 29 March 2021.)

(C) 2021 the authors. This is an open access article under the CC BY (International 4.0) license (www.creativecommons.org/licenses/by/4.0/).

\begin{abstract}
Let $A_{\eta}:=\left\{\left\lfloor 2^{n} \eta\right\rfloor: n=0,1,2, \ldots\right\}$, where $1 \leq \eta<2$. In the recent paper [Discrete Math. Lett. 4 (2020) 31-36], the authors examined the subset-sums of the set $A_{\eta}$ and then presented an encryption algorithm. The basics of the latter part is that the encoded message is a public natural number $N$ and everyone is allowed to query for the set $\left(A_{\eta} \dot{+} A_{\eta}\right) \cap[1, N]$ until they find an element of it. Using the results about $P\left(A_{\eta}\right)$, the set of subset-sums of $A_{\eta}$, it was shown that with the secret key $\gamma$ the message can be decoded in logarithmic time, but for an eavesdropper without the key it takes on average more than $N / \log ^{2} N$ time. In this article, we show that the eavesdropper essentially can not figure out the codeword from the found element of $S$, even if she got that element from the query sequence in a relatively short time.
\end{abstract}

Keywords: subset-sums; Cantor's representation of integers; encoding a codeword.

2020 Mathematics Subject Classification: 11B30, 11B75.

\section{Introduction}

The set $\left\{\left\lfloor 2^{n} \eta\right\rfloor \mid n=0,1, \cdots\right\}$, where $1 \leq \eta<2$, and its related subset-sums were investigated by Erdős and Graham in [4]. Hegyvári also examined some properties of these subset-sums in the articles [2,3]. Based on these works, in the article [1], an encryption algorithm was examined using the aforementioned set and its subset-sums. In this article, we further investigate that encryption process. We show that if an eavesdropper catches the message, she can not be certain what the codeword was.

For any $\eta \in[1,2)$, let $a_{n}:=\left\lfloor 2^{n} \eta\right\rfloor$ and $A_{\eta}:=\left\{a_{n} \mid n=0,1,2, \cdots\right\}$. We associate to $A_{\eta}$ its so-called subset-sums:

$$
P\left(A_{\eta}\right):=\left\{\sum_{i=0}^{\infty} \varepsilon_{i} a_{i}: a_{i} \in A_{\eta} ; \varepsilon_{i} \in\{0,1\} \text { for all } i ; \sum_{i} \varepsilon_{i}<\infty\right\} .
$$

It was proven in the article [1] that every element of $P\left(A_{\eta}\right)$ has a unique representation (see Proposition 3.2 in [1]). It was also shown in [1] (see Proposition 3.3 in [1]) that the biggest gap of the set $P\left(A_{\eta}\right) \cap\left[1, a_{k}\right]$ is equal to $\sum_{j=1}^{k} \eta_{j}$, where $\eta=1 . \eta_{1} \eta_{2} \cdots$ in binary form. Using these results, the authors of [1] also presented an encryption algorithm to send a message. The coding process is given as follows:

Let $c_{n}$ be a binary codeword with $n$ digits: $c_{n}=\eta_{1} \eta_{2} \cdots \eta_{n}$, where each $\eta_{i} \in\{0,1\}$ for $i=1,2, \cdots, n$. We associate an $\eta \in[1,2)$ for the codeword $c_{n}: \eta=1 . \eta_{1} \eta_{2} \cdots \eta_{n} \cdots$ (after $\eta_{n}$ we can extend arbitrarily, only one assumption is important, that we prefer the expression where $\eta_{i}=0$ holds infinitely many times). The message is sent by Alice to Bob in the form of $N \in \mathbb{N}$. This $N$ is public in the sense that an eavesdropper (Eve) can catch this message. Alice and Bob on the other hand has a secret key $0<\gamma<1$, which is available only for them.

Denote by $B+C$ the restricted sum for the sets $B, C \subseteq \mathbb{N}$, i.e.,

$$
B \dot{+} C:=\{b+c \mid b \in B, c \in C \text { and } b \neq c\} .
$$

Towards the decoding process, let $S$ be the following set:

$$
S:=\left(A_{\eta}+A_{\eta}\right) \cap[1, N] .
$$

For the decoding process, let us define a query function $f:[1, N] \mapsto\{0,1\}$ such that $f(x)=0$ if $x \notin S$ and $f(x)=1$ if $x \in S$. Everyone can query an $\left(x_{0}, x_{0}+1, \cdots x_{0}+L\right)$ sequence of integers such that $\left(f\left(x_{0}\right), f\left(x_{0}+1\right), \cdots, f\left(x_{0}+L\right)\right)=(0,0, \cdots, 0,1)$. So we can query $x_{0}$ and if it is not in $S$ we can query $x_{0}+1$ and so on until we find an element of $S$. The length of the query sequence is $L_{\eta}^{x_{0}}(N):=L$. For simplicity, let us denote by $x_{L}$ the number $x_{0}+L$.

In the 4th section of the article [1], the following two results were presented about this process. 
Theorem 1.1. If Alice sends the message $N$ for which it holds that

$$
\gamma N \in\left[\sum_{i=0}^{n-1} a_{i}+1, a_{n}\right),
$$

then Bob can get the message by asking a query sequence starting by an appropriately chosen $x_{0}$ such that

$$
L_{\eta}^{x_{0}}(N) \leq \log _{2} N+2 .
$$

Theorem 1.2. The expected length of the query sequence of an eavesdropper Eve, who chooses the start of the query sequence uniformly at random in $[1, N]$ is

$$
\mathbb{E}(X) \geq \frac{c N}{\log _{2}^{2} N},
$$

$(c>0$ absolute)

\section{Decomposition lemmas}

As we already mentioned, our purpose is to show that Eve can not really recognize the codeword even if she finds an $x_{L} \in S$ in a relatively short time. We are going to restrict the length of a query sequence by $N^{\beta}, 0<\beta<1$. This is not going to cause problems, because this restriction only makes things harder for Eve, while Bob will still have enough query questions to find the codeword in a short time (see Theorem 1.1). We will return to the right choice of $\beta$ later.

Assume now that Eve finds an element $x_{L} \in S$ after querying at most $N^{\beta}$. What she can do is try to decompose $x_{L}$ into the sum of $b$ and $b^{\prime}$, where $b, b^{\prime} \in A_{\zeta}$ (for some $1 \leq \zeta<2$ ) and she hopes that eventually $\zeta=\eta$. This is equivalent to the task of finding $b$ and $b^{\prime}, b+b^{\prime}=x_{L}, b>b^{\prime}$ where in binary form the first few digits of $b$ forms exactly $b^{\prime}$, since both of them are equal to $\left\lfloor 2^{s} \zeta\right\rfloor$ for some $s \in \mathbb{N}$.

Now, we are going to look at this task in a third equivalent form. We search for the pairs $(b, k)$ that satisfy:

$$
x_{L}=b+\left\lfloor\frac{b}{2^{k}}\right\rfloor
$$

where $1<b<x_{L}$ and $1 \leq k \leq\left\lfloor\log _{2} x_{L}\right\rfloor+1$. Notice that $k$ determines $b$, since $x_{L}$ is fixed. Now, our goal is to guarantee that for many $k$ such $b$ exists. For this, lets analyze (1) for a fixed $k$.

Lemma 2.1. For fixed $x_{L}, k \in \mathbb{N}$, there is a solution for (1) if and only if $x_{L} \not \equiv-1 \bmod \left(2^{k}+1\right)$.

Proof. First lets reshape (1):

$$
\begin{gathered}
x_{L}=b+\left\lfloor\frac{b}{2^{k}}\right\rfloor \Longleftrightarrow b+\frac{b}{2^{k}}-1<x_{L} \text { and } x_{L} \leq b+\frac{b}{2^{k}} \Longleftrightarrow \\
\Longleftrightarrow b \frac{2^{k}+1}{2^{k}}<x_{L}+1 \text { and } x_{L} \leq b \frac{2^{k}+1}{2^{k}} \Longleftrightarrow \frac{2^{k}}{2^{k}+1} x_{L} \leq b<\frac{2^{k}}{2^{k}+1}\left(x_{L}+1\right) .
\end{gathered}
$$

To guarantee a solution to (1), we need to prove the existence of an integer greater than

but strictly less than

$$
\frac{2^{k} x_{L}}{2^{k}+1}
$$

$$
\frac{2^{k} x_{L}+2^{k}}{2^{k}+1}
$$

We claim that there is such an integer if and only if $x_{L} \not \equiv-1 \bmod \left(2^{k}+1\right)$. Lets look at $2^{k} x_{L}$ in the following form:

$$
2^{k} x_{L}=p_{k}\left(2^{k}+1\right)+t_{k},
$$

where $0 \leq t_{k} \leq 2^{k}, t_{k} \in \mathbb{Z}$. We need an integer

$$
b^{\prime} \in\left[\frac{2^{k} x_{L}}{2^{k}+1}, \frac{2^{k} x_{L}+2^{k}}{2^{k}+1}\right)=\left[p_{k}+\frac{t_{k}}{2^{k}+1}, p_{k}+\frac{t_{k}+2^{k}}{2^{k}+1}\right) .
$$

This is equivalent to the existence of an integer

$$
b^{\prime \prime} \in\left[\frac{t_{k}}{2^{k}+1}, \frac{t_{k}+2^{k}}{2^{k}+1}\right) .
$$

If $t_{k}=0$ then $0 \in\left[\frac{t_{k}}{2^{k}+1}, \frac{t_{k}+2^{k}}{2^{k}+1}\right)$, and if $t_{k}>1$ then $1 \in\left[\frac{t_{k}}{2^{k}+1}, \frac{t_{k}+2^{k}}{2^{k}+1}\right)$. It follows that there is such an integer if and only if

$$
t_{k} \neq 1 \Longleftrightarrow 2^{k} x_{L} \not \equiv 1 \quad \bmod \left(2^{k}+1\right) \Longleftrightarrow x_{L} \not \equiv-1 \quad \bmod \left(2^{k}+1\right) \text {. }
$$


Our purpose now is to show the existence of many $k$, where this incongruence holds. The following lemma will ensure this.

Lemma 2.2. Let $k$ be an integer such that $k \geq \frac{1}{2} \log _{2} x_{L}$. In this case, there is an integer solution for (1) either for $k$ or for $k+1$.

Proof. For contradiction, suppose that both of the following linear congruences hold:

$$
x_{L} \equiv-1 \quad \bmod \left(2^{k}+1\right) \text { and } x_{L} \equiv-1 \quad \bmod \left(2^{k+1}+1\right) .
$$

On the other hand, $2^{k}+1$ and $2^{k+1}+1$ are relative primes, so by the Chinese remainder theorem any solution to the above congruent system has a unique solution under $\bmod \left(2^{k}+1\right)\left(2^{k+1}+1\right)$. Hence, the smallest positive integer that satisfies both of the above congruences simultaneously is $\left(2^{k}+1\right)\left(2^{k+1}+1\right)-1$. Thus $x_{L} \geq\left(2^{k}+1\right)\left(2^{k+1}+1\right)-1$. From this, a trivial estimate shows that $x_{L}>2^{2 k}$, contradicting the assumptions of the theorem.

\section{Result on security against an eavesdropper}

Now, we want to underestimate the number of $b$ 's which Eve considers viable. From the previous lemma, we know that Eve can decompose $x_{L}$ in many ways to satisfy (1). But, to delude Eve it is not enough to just have $b$ 's which satisfy (1). Remember that Eve wants $b$ 's which can be the starting sequence of the codeword and she can check if this hypothesis of her is compatible with the queries or not. What do we mean by this?

Fix a $b \in \mathbb{N}$ such that for some $k: x_{L}=b+\left\lfloor\frac{b}{2^{k}}\right\rfloor$. Considering Eve's point of view, she thinks that she queried from a set $A_{\zeta}+A_{\zeta}$, where $\zeta \in[1,2)$ and she hopes that $\zeta=\eta$. To have this theory, she has to assume that $a_{j}=\left\lfloor 2^{j} \zeta\right\rfloor=b$, where $j=\left\lfloor\log _{2} b\right\rfloor$. In this case, the first elements of the set $A_{\zeta}$ would be:

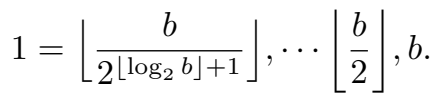

Denote by $C_{b}$ the set that has exactly the same elements as above:

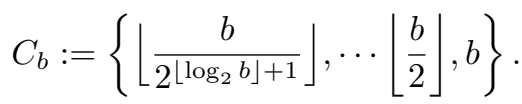

Eve can exclude certain $b$ 's by looking up if during her query sequence she queried for some $x \in A_{\zeta} \dot{+} A_{\zeta}, x<x_{L}$. She can do it because if she did indeed query such an $x$, then she would have got $f(x)=1$. But, in this case she would have stopped querying there and would have got $x$ as an element of $S$ instead of $x_{L}$.

Before we go further, we prove a simple statement that ensures that Eve actually knows $\left(A_{\zeta} \dot{+} A_{\zeta}\right) \cap\left[1, x_{L}\right]$. Or in other words, the exclusion method relies only on $b$ and not on the elements of $A_{\zeta}$, larger than $b$ (which are unknown to Eve).

Claim. $\left(A_{\zeta}+A_{\zeta}\right) \cap\left[1, x_{L}\right]=\left(C_{b} \dot{+} C_{b}\right) \cap\left[1, x_{L}\right]$.

Proof. First, we recall that we assume $b=\left\lfloor 2^{j} \zeta\right\rfloor=a_{j}$. It is obvious that $\left(A_{\zeta} \dot{+} A_{\zeta}\right) \cap\left[1, x_{L}\right] \supseteq\left(C_{b} \dot{+} C_{b}\right) \cap\left[1, x_{L}\right]$. For the other direction, take an arbitrary $x \in\left(A_{\zeta} \dot{+} A_{\zeta}\right) \cap\left[1, x_{L}\right]$. Notice that it is enough to prove that $x=a_{l}+a_{m}$ holds for some $l, m \leq j, l \neq m$. We know by assumption that $x_{L}=a_{j}+a_{k}$ for some $k<j$. Note that for every $n \in \mathbb{N}: 2 a_{n}=2\left\lfloor 2^{n} \zeta\right\rfloor \leq$ $\left\lfloor 2^{n+1} \zeta\right\rfloor=a_{n+1}$. Assume for contradiction that $x=a_{l}+a_{m}$ and $l>j$, in this case we would get $x_{L}=a_{j}+a_{k}<2 a_{j} \leq a_{j+1} \leq$ $a_{l}<a_{l}+a_{m}=x$.

We are going to say that a $b$ is acceptable for Eve if it is a solution of (1) for some $k$ and it can not be excluded by Eve with the previous method (checking if she queried for any $x<x_{L}, x \in A_{\zeta} \dot{+} A_{\zeta}$, which is equivalent, by the above claim, to checking if she queried for any $x<x_{L}, x \in C_{b}+C_{b}$ ). We want to emphasize here, that acceptability now relies only on $b$ (and not on the elements of $A_{\zeta}$ which are larger than $b$ ). Lets denote the number of acceptable solution for Eve by $\mu$ (for a fixed $\left.x_{L}\right)$.

We want Eve to have a lot of acceptable b's so we try to deny the ability of exclusion from her. For this purpose, from now on we restrict the length of the query sequence by $N^{\beta}$. How does this affect Eve's exclusion method? First notice that in order to exclude a certain $b$ it is enough for her to check if she queried $\hat{x}=\max \left\{x \in C_{b}+C_{b} \mid x<x_{L}\right\}$, the largest element of $C_{b}+C_{b}$ smaller than $x_{L}$. So if we can ensure that $N^{\beta} \leq x_{L}-\hat{x}$, then she can not exclude this $b$. We will use this observation in the next theorem.

As we mentioned earlier, we are proving that Eve can not figure out the codeword uniquely. We need to specify what that means exactly. So far we have investigated only solutions for (1). If Eve finds a $k$ and a corresponding $b$ as a solution 
of (1), then she assumes $b=\left\lfloor 2^{s} \zeta\right\rfloor$ for some $s \in \mathbb{N}, 1 \leq \zeta<2$ and hopes that $\zeta=\eta$. The first $n+1$ digits of $\zeta$ is equivalent to an $n$ digit codeword $\left(1 . \zeta_{1} \zeta_{2} \cdots \zeta_{n} \cdots \sim d=\zeta_{1} \zeta_{2} \cdots \zeta_{n}\right)$, so she has to get these $n+1$ digits. Since the length of $b$ is at most the length of $x_{L}$, Eve only knows at most $\left\lfloor\log _{2} x_{L}\right\rfloor+1$ digits of $\zeta$. So she can choose the values of the remaining $h=n+1-\left(\left\lfloor\log _{2} x_{L}\right\rfloor+1\right)=n-\left\lfloor\log _{2} x_{L}\right\rfloor$ digits arbitrarily, leaving her with at least $2^{h}$ different $\zeta$, which have the property, that their first $\left\lfloor\log _{2} b\right\rfloor+1$ digits form the number $b$. And this is only for one acceptable $b$. (We know that at least one always exists, which is the one corresponding to $\eta$, which is determined by the real codeword.)

So if $x_{L}$ is too small, then this leaves Eve with a lot of unknown information about the codeword. The second part of the next theorem is about the case when she finds an $x_{L}$ where $n-\left\lfloor\log _{2} x_{L}\right\rfloor$ is small (i.e. $x_{L}$ is large). In this case there are many acceptable ways to decompose $x_{L}$. So she has many $b$ 's to start the codeword with, leaving her in inconsistency, even though she has low level of freedom to extend them into possible codewords.

We are going to call an integer $d=\zeta_{1} \zeta_{2} \cdots \zeta_{n}$ (where each $\zeta_{i} \in\{0,1\}$ for $i=1,2, \cdots, n$ ) possible codeword viable if there is an acceptable $b$ which is the prefix of $d$ in binary form. For a fixed $x_{L}$ denote the number of viable codewords by $\nu$. Obviously $\mu \leq \nu$, since every acceptable $b$ can be extended (possibly in many ways) into a viable $d$.

In the upcoming theorem we will consider two cases, $x_{L} \geq N^{\alpha}$ or $x_{L} \leq N^{\alpha}$, where $0<\alpha<1$, and we will give a lower bound on $\mu$ and $\nu$ respectively. We will give some more restriction on $\alpha$ later and we will also discuss its relationship to other variables $N, \gamma, \beta$.

Theorem 3.1. (i). If $x_{L}<N^{\alpha}$ then

$$
\nu \geq \frac{\gamma N^{1-\alpha}}{2}
$$

(ii). If $x_{L} \geq N^{\alpha}$ then

$$
\mu \geq \frac{\left(\alpha-\beta-\frac{2}{\log _{2} N}-\frac{1}{2}\right) \alpha \log _{2} N-3}{2} .
$$

Proof. (i). In this case, even if Eve finds the correct $b$ for $x_{L}$ she misses some bits of the codeword. To be more precise, if the length of the codeword is $n$ we have the following inequalities:

$$
\text { missing bits }=n-\left\lfloor\log _{2} x_{L}\right\rfloor \geq\left\lfloor\log _{2} \gamma N\right\rfloor-\left\lfloor\log _{2} x_{L}\right\rfloor \geq \log _{2} \gamma-1+\log _{2} N-\log _{2} x_{L} \geq \log _{2} \gamma+(1-\alpha) \log _{2} N-1 .
$$

Thus,

$$
\mu \geq 2^{\text {missing bits }} \geq \frac{\gamma N^{1-\alpha}}{2}
$$

(ii). We are going to prove the existence of acceptable $b$ 's belonging to certain values of $k \in\left[\frac{1}{2} \log _{2} x_{L},\left(\alpha-\beta-\frac{2}{\log _{2} N}\right) \log _{2} x_{L}\right]$. From Lemma 2.2, we know that if $k \geq \frac{1}{2} \log _{2} x_{L}$, then for either $k$ and $k+1$ there is a $b$ solution for (1). But, it also needs to be acceptable. Because of the argument above we only have to ensure $N^{\beta} \leq x_{L}-\hat{x}$ for this.

Since $x_{L}=b+\left\lfloor\frac{b}{2^{k}}\right\rfloor, \hat{x}$ is either $b+\left\lfloor\frac{b}{2^{k+1}}\right\rfloor$ or $\left\lfloor\frac{b}{2}\right\rfloor+\left\lfloor\frac{b}{4}\right\rfloor$ and therefore

$$
\left\lfloor\frac{b}{2^{k}}\right\rfloor-\left\lfloor\frac{b}{2^{k+1}}\right\rfloor \leq \min \left(\left\lfloor\frac{b}{2^{k}}\right\rfloor-\left\lfloor\frac{b}{2^{k+1}}\right\rfloor, b+1-\left\lfloor\frac{b}{2}\right\rfloor-\left\lfloor\frac{b}{4}\right\rfloor\right) \leq x_{L}-\hat{x} .
$$

So, we are left to see if the following inequality holds:

$$
\left\lfloor\frac{b}{2^{k}}\right\rfloor-\left\lfloor\frac{b}{2^{k+1}}\right\rfloor \geq N^{\beta} .
$$

Starting from the left side we get:

$$
\left\lfloor\frac{b}{2^{k}}\right\rfloor-\left\lfloor\frac{b}{2^{k+1}}\right\rfloor \geq \frac{b}{2^{k}}-1-\frac{b}{2^{k+1}} \geq \frac{x_{L}}{2^{k+2}} \geq \frac{N^{\alpha}}{2^{k+2}} .
$$

So, we need $\frac{N^{\alpha}}{2^{k+2}} \geq N^{\beta}$. Since we are now considering $k \leq\left(\alpha-\beta-\frac{2}{\log _{2} N}\right) \log _{2} x_{L}$, we get the following:

$$
k \leq\left(\alpha-\beta-\frac{2}{\log _{2} N}\right) \log _{2} x_{L} \leq\left(\alpha-\beta-\frac{2}{\log _{2} N}\right) \log _{2} N=\log _{2} N(\alpha-\beta)-2 .
$$

If we look at the left and right side and reorder the inequality we can easily get the desired inequality. Now, from this and from Lemma 2.2 we can say that for every $k$ in the interval $\left[\frac{1}{2} \log _{2} x_{L},\left(\alpha-\beta-\frac{2}{\log _{2} N}\right) \log _{2} x_{L}\right]$ either $k$ or $k+1$ gives an acceptable $b$ for Eve. So, by pairing the integers of interval we get

$$
\begin{gathered}
\mu \geq\left\lfloor\frac{\left\lfloor\left(\alpha-\beta-\frac{2}{\log _{2} N}\right) \log _{2} x_{L}\right\rfloor-\left\lceil\frac{1}{2} \log _{2} x_{L}\right\rceil+1}{2}\right\rfloor \geq\left\lfloor\frac{\left(\alpha-\beta-\frac{2}{\log _{2} N}\right) \log _{2} x_{L}-\frac{1}{2} \log _{2} x_{L}-1}{2}\right\rfloor \geq \\
\geq \frac{\left(\alpha-\beta-\frac{2}{\log _{2} N}-\frac{1}{2}\right) \log _{2} x_{L}-3}{2} \geq \frac{\left(\alpha-\beta-\frac{2}{\log _{2} N}-\frac{1}{2}\right) \alpha \log _{2} N-3}{2} .
\end{gathered}
$$




\section{Concluding remarks}

It is worth to take a look at what the results of Theorem 3.1 really mean. In both parts of this theorem, we get more acceptable solutions if $N$ is large. We can guarantee this part, but after a certain point an absurdly large $N$ is not very practical. So, we want to optimize the parameters $\alpha$ and $\beta$ as well.

The parameter $\beta$ is best to be as small as possible (but can not be too small because Bob has to be able to find the codeword). Theorem 1.2 tells us that the lower bound $\log _{2} N+1<N^{\beta}$ is required to give Bob the ability to get the codeword with the secret key $\gamma$, regardless of the restriction on the length of a query sequence. So, we can choose for example $\beta=\log _{N}(2 \log N)$, which tends to 0 , as $N \rightarrow \infty$.

In the case of $\alpha$, it is a bit more complicated than that $\beta$. We can say that $\alpha$ is a double-edged weapon in the following sense: According to part (i), $\alpha$ is better to be small, while according to part (ii) it is better to be large (close to 1).

We gather here the acquired bounds on $N$ with respect to the parameters. Here, we consider the case when we only want to ensure more than one viable codeword. From Theorem 3.1, we get that if we want $\nu>1$ in part (i) and $\mu>1$ in part (ii), then $N$ has to satisfy the following lower bounds: (i) $\rightarrow N>\left(\frac{2}{\gamma}\right)^{\frac{1}{1-\alpha}}$ and (ii) $\rightarrow N>2^{\frac{7}{\alpha(\alpha-\beta-1 / 2)}}$.

\section{Acknowledgment}

The authors are supported by the European Union, co-financed by the European Social Fund (EFOP-3.6.3-VEKOP-162017-00002).

\section{References}

[1] B. Bakos, N. Hegyvári, M. Pálfy, X. H. Yan, On subset sums of pseudo-recursive sequences, Discrete Math. Lett. 4 (2020) 31-36.

[2] N. Hegyvári, Some remarks on a problem of Erdős and Graham, Acta Math. Hungar. 53 (1989) 149-154.

[3] N. Hegyvári, Subset sums in $\mathbb{N}^{2}$, Combin. Probab. Comput. 5 (1996) 393-402.

[4] P. Erdős, R. L. Graham, Old and new problems and results in combinatorial number theory: van der Waerden's theorem and related topics, Enseign. Math. 25 (1979) 325-344. 